\title{
Molecular Dynamics Study of Iron-Nickel Alloys
}

\author{
R. Meyer and P. Entel \\ Theoretische Tieftemperaturphysik, Gerhard-Mercator-Universität, Gesamthochschule Duisburg, \\ Lotharstrasse 1, 47048 Duisburg, Germany
}

\begin{abstract}
We present results of molecular dynamics simulations of disordered iron-nickel alloys. In particular we discuss the $\alpha-\gamma$ transition and associated anharmonic properties by making use of semiempirical potentials. Our results show that the structural transformation in these alloys is driven by local disorder. From data of specific heat and thermal expansion we conclude that the lattice dynamics at elevated temperatures (above the Curie temperature) can be described correctly without considering short range magnetic effects.
\end{abstract}

\section{INTRODUCTION}

Structural phase transitions are a very common phenomena of metallic alloys. While many nonmagnetic compounds show a transition from an open structure like bcc at high temperatures to a close-packed structure, e.g. fcc, in the low-temperature regime, many magnetic alloys undergo a transition into the opposite direction. Iron-rich FeNi alloys show a transition from fec to bcc with decreasing temperature which is accompanied by the onset of magnetic order. From experiments it is known that in these disordered magnetic alloys the martensitic transformation exhibits a very large temperature hysteresis. Other features of these systems are associated with the Invar effect in alloys containing 35 at \% nickel and the simultaneous occurence of premartensitic behaviour without a structural change. All these phenomena seem to be connected with large anharmonicites [1].

In this paper we present results from molecular dynamics simulations of FeNi-alloys, showing that it is possible to describe the properties of these materials above the Curie temperature and the structural change without the necessity of including explicit magnetic exchange terms.

\section{COMPUTATIONAL METHODS}

With increase of computer power atomistic simulations have become a more and more important tool in the investigation of material properties. While $a b$-initio methods like the Car-Parinello scheme [2] or tight-binding molecular dynamics [3] are still limited to very small system sizes, (semi-)empirical methods allow nowadays to handle thousands to millions of atoms. We have done molecular dynamics simulations of disordered iron-nickel alloys whereby the interatomic forces have been calculated by using the embedded-atom method (EAM). This method is based on density-functional theory and has been introduced by Daw and Baskes $[4,5]$. It is assumed that each atom $j$ adds a spherically averaged electron density $\rho_{j}^{\text {at }}$ to the whole (valence-) electron density in the system. Then the contribution of atom $i$ to the potential energy $E$ of the system is a function of the type of the atom and the 
local electron density provided by the surrounding atoms at the position $\mathbf{r}_{i}$ of the atom $i$. In order to account for short-range repulsive interactions of the ions, a pair-potential has been added to the potential energy. The total energy $E$ is then given by

$$
E=\sum_{i} F_{i}\left(\rho_{i}\right)+\frac{1}{2} \sum_{i \neq j} \Phi_{i j}\left(r_{i j}\right)
$$

with

$$
\rho_{i}=\sum_{i \neq j} \rho_{j}^{\text {at }}\left(r_{i j}\right)
$$

$\left(r_{i j}\right.$ denotes the distance between atoms $i$ and $j$ ). The pair-potential $\Phi_{i j}$ is assumed to be of Coulomb form

$$
\Phi_{i j}\left(r_{i j}\right)=\frac{Z_{i}\left(r_{i j}\right) Z_{j}\left(r_{i j}\right)}{r_{i j}} .
$$

The functions $F$ and $Z$ which we have used in our simulations, were obtained by fitting cubic splines to experimental data of the lattice constant, cohesive energy, elastic constants, vacancy-formation energy and some zone-boundary phonon frequencies of pure iron and nickel. The $Z$ functions have been forced to go to zero with vanishing derivative between second and third neighbours. The use of elastic constants and zone-boundary frequencies in the fitting procedure guarantees a good overall description of the phonon spectra of the elements, and hence the correct harmonic part of the lattice dynamics. The vacancy-formation energy adjusts to a certain degree to the anharmonic behaviour of the metals. Following Daw and Baskes the electron density provided by an atom is the sum of a $4 \mathrm{~s}$ and a $3 \mathrm{~d}$ contribution

$$
\rho_{j}^{\mathrm{at}}\left(r_{i j}\right)=n_{4 s}\left|\Psi_{i}^{4 s}\right|^{2}+n_{3 d}\left|\Psi_{i}^{3 d}\right|^{2} .
$$

In our calculations we have taken the wave-functions $\Psi^{4 s}$ and $\Psi^{3 d}$ for iron and nickel from Clementi and Roetti [6] with an applied constant shift to force the electron density being zero at a cutoff distance between third and fourth nearest neighbours. The effective number of $4 \mathrm{~s}$ electrons $n_{4 s}$ has been obtained by a fit procedure which gives the lattice constant of $\mathrm{Fe}_{3} \mathrm{Ni}$. The number of $3 \mathrm{~d}$ electrons was taken as the difference $\left(n_{\mathrm{val}}-n_{4 \mathrm{~s}}\right)$ between the number of valence electrons $n_{\mathrm{val}}$ and $n_{4 s}$.

With these potentials we performed molecular dynamics simulations using the well known Verlet algorithm with a time step of $\tau=1.510^{-15} \mathrm{~S}$ and the Parinello-Rahman scheme [7] allowing for a fluctuating shape of the simulation box and hence for a structural transition. Our system size is 1024 atoms with different configurations and concentrations of nickel starting with a perfect bcc lattice at low temperatures. After an equilibration phase of 1000 time steps at $300 \mathrm{~K}$ for each configuration, runs were performed at temperatures up to $1200 \mathrm{~K}$ doing 5000 to 10000 time steps at each temperature. The temperature was controlled by the Nosé-thermostat method [8]. We also made some runs starting with an fcc lattice at high temperatures and cooling slowly down. All calculations have been done at zero pressure.

\section{RESULTS AND DISCUSSION}

Fig. 1 shows the distance-distribution function which we obtained for the $\mathrm{Fe}_{65} \mathrm{Ni}_{35}$ alloy at temperatures $T=300 \mathrm{~K}$ and $600 \mathrm{~K}$. The peaks correspond to a bcc structure with lattice constant $2.83 \AA$. At $600 \mathrm{~K}$ the distribution function shows indications of a fcc structure with an unsual broad fourth nearest neighbour peak which is shifted to lower distances. This is probably due to an unperfect transition which leads to large distortions. Nevertheless we have successfully checked that indeed an fcc structure is present in the alloy by looking directly at the three-dimensional picture of the thermally averaged particle positions. At temperatures higher than $600 \mathrm{~K}$ the distribution function does not show any further principal changes. So we can conclude that the distortions do not vanish 


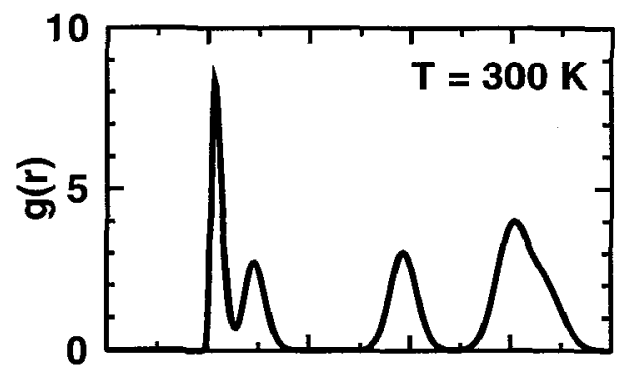

Fig. 1: Distance-distribution function of $\mathrm{Fe}_{65} \mathrm{Ni}_{35}$. The upper graph shows the function obtained at $T=300 \mathrm{~K}$ in the $\alpha$-phase. The lower graph showes the distribution in the $\gamma$-phase at $T=600 \mathrm{~K}$.

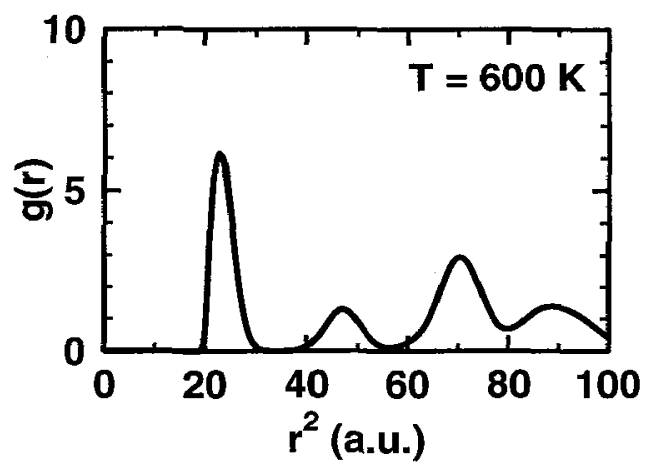

and are probably related to the periodic boundary conditions in connection with the small system size. For other nickel concentrations the behaviour is rather similar, except for $\mathrm{Fe}_{90} \mathrm{Ni}_{10}$, where the change of the simulation box is different and less distortions occur. For pure iron so far no transition could be observed. This leads us to the assumption that the structural transition in the alloys is driven by local disorder and that its mechanism might be different from the $\alpha-\gamma$ transition in pure iron.

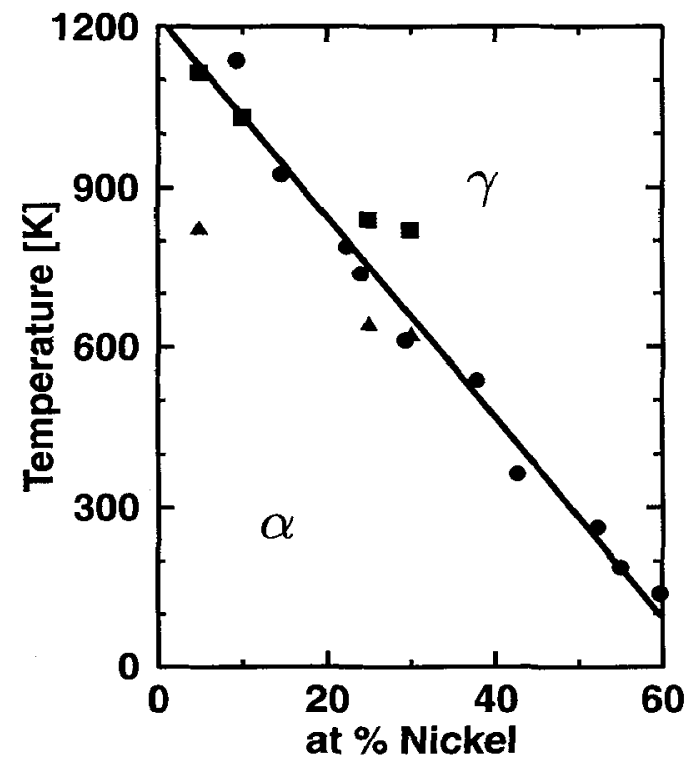

Fig. 2: Phase diagram of the $\alpha-\gamma$ transition. Circles indicate the transition temperatures derived from molecular dynamics simulations. The solid line is the result of a linear fit to these data. The experimental results for the transition temperatures are shown by the triangles $\left(A_{s}\right)$ and squares $\left(A_{f}\right)[9]$. 


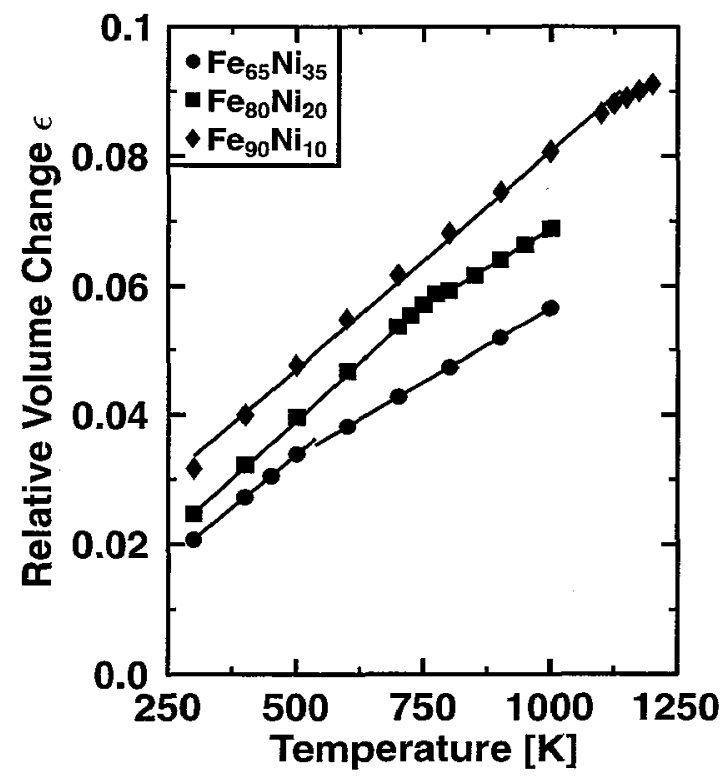

Fig. 3: Relative volume change of $\mathrm{Fe}_{65} \mathrm{Ni}_{35}$, $\mathrm{Fe}_{80} \mathrm{Ni}_{20}$, and $\mathrm{Fe}_{90} \mathrm{Ni}_{10}$ as a function of temperature. The lines are the results of a linear least-square fit to the data points.

The phase diagram of the $\alpha-\gamma$ transition which follows from our calculations, is shown in Fig. 2 . The transition temperatures are in general too high but are of the same order as the experimental values. It can be seen from Fig. 2 that the transition follows a straight line as a function of nickel concentration with deviations at both ends. This behaviour is in accordance with experimental findings. Unfortunately we could not observe a transition from fcc to bcc by cooling down the system. Therefore, the following picture emerges: The potentials used by us favour a structural transition yet hysteresis already being large in the real system, is overestimated in the simulations. There are two reasons for this behaviour. On the one hand, the small system sizes hinder a local transformation with long-ranged relaxations. This can probably not be checked by increasing the system size because it would be necessary to go to mesoscopic system sizes which currently can not be handled. On the other hand, we have fitted the potentials to the magnetic ground-state properties of iron and nickel. Since the transition experimentally takes place above the Curie temperature, the lack of magnetic interactions probably lowers the transition temperature significantly. It would have been better to fit the potentials to experimental properties in the nonmagnetic state. However, it is a very difficult task to fit the potentials at finite temperatures. Work to include magnetism into the simulation procedure is in progress but so far we have no results.

A rather critical point in the description of lattice dynamics at higher temperatures is the anharmonic contribution to the interactions which are not included in the phonon dispersion curves. One quantity strongly influenced by anharmonicities is the thermal expansion coefficient $\alpha$. In order to show the behaviour of this coefficient we have drawn in Fig. 3 the relative volume change of our systems for concentrations of 10,20 and 35 atomic percent nickel. The reference volume is that of the relaxed ground-state structure. The structural transition can easily be identified by the steps in the data. The slopes of the regression lines lead to expansion coefficients $15.310^{-6} \mathrm{~K}^{-1}$ for $\mathrm{Fe}_{65} \mathrm{Ni}_{35}$ and $15.910^{-6} \mathrm{~K}^{-1}$ for $\mathrm{Fe}_{80} \mathrm{Ni}_{20}$ in the fcc structure, and $22.510^{-6} \mathrm{~K}^{-1}$ for $\mathrm{Fe}_{90} \mathrm{Ni}_{10}$ in the bcc structure. These values are very close to experimental results [10].

Another quantity influenced by anharmonicities is the specific heat at constant pressure $c_{p}$. Therfore, we show in Fig. 4 the mean enthalpy per atom for the same systems used in Fig. 3. As in Fig. 3 the phase transition leads to steps in the otherwise linearly behaving data. The fact that the enthalpy is obviousiy not raising at the transition, indicates that the system is not in equilibrium but that the transition is overheated. The slopes of the regression lines lead to values of $c_{p}$ slightly 


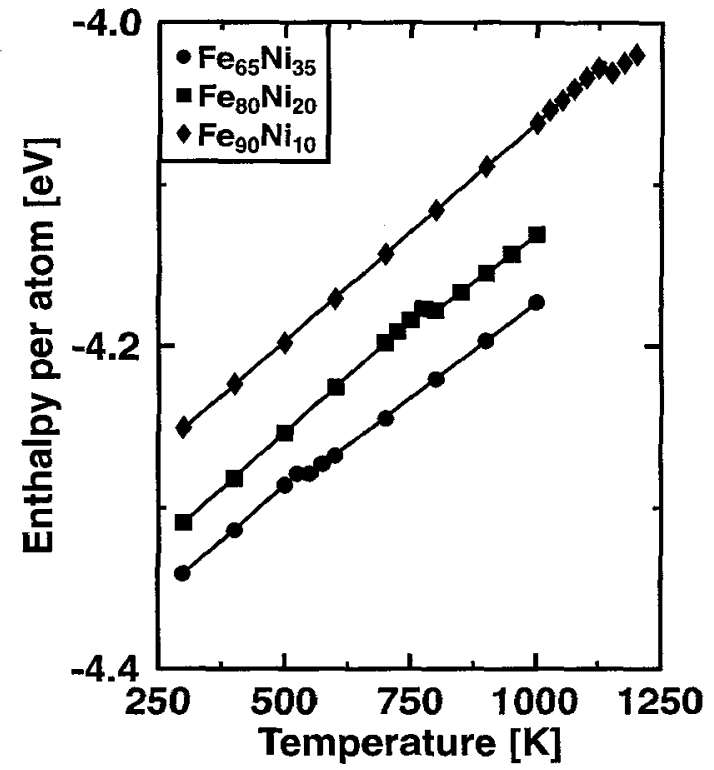

Fig. 4: Enthalpy per atom of $\mathrm{Fe}_{65} \mathrm{Ni}_{35}$, $\mathrm{Fe}_{80} \mathrm{Ni}_{20}$, and $\mathrm{Fe}_{90} \mathrm{Ni}_{10}$ as a function of temperature. The lines show the results of linear least-square fits to the data points.

below $3 \mathrm{R}$ for the systems with fcc structure and slightly above $3 \mathrm{R}$ for the bcc structure. This is also in accordance with experimental findings [11] if one would take into account the missing magnetic terms.

From our simulations we can conclude that the anharmonic behaviour of the disordered ironnickel alloys is quiet well reproduced by our potentials without the explicit inclusion of magnetism via exchange terms. The structural transformation in these systems is clearly reproduced by our calculations. Yet the correct experimental transition temperatures can probably not be obtained as long as we do not consider the influence of magnetism on anharmonic effects.

In near future we plan, besides the inclusion of magnetism, to look for temperature-induced changes in the phonon-spectra. Therefore, simulations with fixed volume are under work because dynamical properties like the phonon-spectral intensity cannot be correctly calculated by using the Parinello-Rahman scheme. The results of these simulations will be the subject of a forthcoming paper.

\section{ACKNOWLEDGEMENT}

This work has been supported by the Sonderforschungsbereich 166 of the DFG.

\section{References}

[1] Hoffmann E., Herper H., Entel P., Mishra S.G., Mohn P., Schwarz K., Phys. Rev. B 47 (1992) 5589-5596.

[2] Car R., Parrinello M., Phys. Rev. Lett. 55 (1985) 2471-2474.

[3] Wang C.Z., Chan C.T. and Ho K.M., Phys. Rev. B 39 (1988) 8586-8592.

[4] Daw M.S. and Baskes, M.I., Phys. Rev. Lett 50 (1983) 1285-1288.

[5] Daw M.S. and Baskes, M.I., Phys. Rev. B 29 (1984) 6443-6453. 
[6] Clementi E., Roetti C. Atomic Data and Nuclear Data Tables (Academic, New York, 1974), Vol. 14. Nos. 3 and 4.

[7] Parrinello M., Rahman A., Phys. Rev. Lett. 45 (1980) 1196-1199.

[8] Shuichi Nosé, "Molecular dynamics simulations at constant temperature and pressurc", in "Computer Simulations in Material Science", ed. by Meyer M. and Pontikis V., Kluwer Academic.

[9] Acet M., Schneider T., Wassermann E., private communication

[10] Acet M., private communication.

[11] Rellinghaus B., private communication. 